\title{
After Xigris, researchers look to new targets to combat sepsis
}

Experimental drug therapies for sepsis have not fared well recently. In January 2011, Japan's Eisai announced that it would delay seeking regulatory approval for the company's antiToll-like receptor 4 compound after the drug, known as eritoran, failed to show any benefit in a pivotal 2,000-person trial. And Eli Lilly famously pulled Xigris (activated protein C) from the market in October following the negative results of the 1,700-person PROWESSSHOCK trial. With the withdrawal of Xigris, critical-care physicians now have no drugs specifically approved to treat severe sepsis. But, after all the latest hard knocks to an already downtrodden field, scientists are picking themselves back up and tackling new approaches. They're studying new drugs and drug targets and focusing their efforts on how to better identify the most at-risk patients with sepsis and design more insightful clinical trials.

"There's this notion of despair among some people now. They say it's too complicated and nothing will ever work," says Steven LaRosa, an infectious disease physician at the Texas A\&M Health Science Center in College Station who was involved in testing both Eisai's and Lilly's failed products. "But that's the wrong mindset. We absolutely are chipping away at sepsis. I think that if we just get more sophisticated at how we do these trials, there will be therapies." And those therapies could be lucrative. According to Santosh Mishra, an analyst with London-Based GlobalData, the worldwide sepsis market was valued at $\$ 5$ billion in 2010 and is expected to grow by $50 \%$ over the next five years. "Significant unmet need is present," he says, "which suggests that there is considerable market share for new entrants to capture in the future."

The difficulty of designing effective sepsis drugs revolves around the complex-and not fully understood-pathophysiology of sepsis. The condition can begin with an infection anywhere in the body and proceed to sepsis when microbes move into the bloodstream and ramp up the innate immune system. A diverse array of immune cells, inflammatory modulators and cytokines are activated or released into the blood. Many of these encourage inflammation or blood clotting, and each represents a potential drug target. At the same time, the adaptive immune system, a second and more specific line of defense, begins to falter, becoming less effective at ridding the body of the pathogen or protecting against future infections.

Therapies currently in the pipeline target each step in this disease progression (see 'Antiseptic swipes'). Starting from the beginning, some companies are developing drugs or devices to remove pathogens from the bloodstream or directly target their ill effects. Toraymyxin, for example, is an antibiotic-coated filter from Toronto-based Spectral Diagnostics currently being tested in 600 people suffering from septic shock across the US, Canada and France. "We are on track with all our predictions for our trial," says Paul Walker, chief executive of Spectral. In an earlier phase 2 study, the perfusion system helped lower 28 -day mortality rates from $53 \%$ to $32 \%$ when added to standard care (JAMA 301, 2445-2452, 2009).

Another strategy blocks the pathogens' so-called superantigens, toxins produced by bacteria that can activate as much as $20 \%$ of the body's T cells at once and may be responsible for launching the sepsis-causing immune reaction in the first place. The Israeli company Atox Bio has one such superantigen antagonist, which mimics part of the T cell co-receptor CD28, currently in phase 2 development.

\section{Immune challenge}

Other drugs in the pipeline aim to dampen the body's innate immune response after sepsis or after septic shock has kicked in. These include ART-123, a recombinant form of the anticoagulant protein thrombomodulin from Japan's Asahi Kasei Pharma currently in phase 3 testing in the US, and talactoferrin alfa, an immunomodulatory lactoferrin from Germany's Agennix that sits in limbo after the company halted a late-stage trial in response to interim data showing that more participants were dying in the treatment arm of the study than in the placebo arm.

Many in the field are also eagerly awaiting the results of a 300-person trial testing AstraZeneca's CytoFab, an antibody directed against tumor necrosis factor-alpha (TNF- $\alpha$ ), a prime mediator of inflammation. Previous experimental TNF- $\alpha$-targeting monoclonal antibodies have failed to show an effect on mortality rates in patients with sepsis. Yet experts predict that CytoFab will fare better because it contains many different antibody fragments, which could lead to a more complete blockage of the cytokine's function. In a 70-person dose-escalation trial published in February, study subjects who received multiple doses of CytoFab had near-undetectable levels of TNF- $\alpha$ in their blood serum within two hours of starting the drug treatment, and they experienced only mild side effects (Crit. Care 16, R31, 2012).

A final set of sepsis drugs are directed at boosting the adaptive immune system response to restore the body's ability to fight the disease. Such drugs-which include interleukin modulators, immunoadjuvants and other ways of controlling $\mathrm{T}$ and $\mathrm{B}$ cell function-are not as far along in clinical development, but their importance is beginning to be underscored by basic research.

"We don't have a magic drug yet," says Shaw Warren, an infectious disease specialist at the Massachusetts General Hospital in Boston. "But we've learned a lot. The field is regrouping now, and people are looking for a new direction."

Sarah C P Williams

\begin{tabular}{|c|c|c|c|c|}
\hline Approach & Drug & Company & Mechanism & Phase \\
\hline \multirow{2}{*}{$\begin{array}{l}\text { Targeting pathogens } \\
\text { and their products }\end{array}$} & Toraymyxin & Spectral Diagnostics & Antibiotic-coated hemoperfusion column & 3 \\
\hline & AB103 & Atox Bio & Blocks bacterial superantigens & 2 \\
\hline \multirow{4}{*}{$\begin{array}{l}\text { Dampening innate immune } \\
\text { and inflammatory responses }\end{array}$} & ART-123 & Asahi Kahei Pharma & Recombinant thrombomodulin & $3^{a}$ \\
\hline & Bicizar & BioGenius & C1-esterase inhibitor blocks complement activation & $3^{b}$ \\
\hline & Actos (pioglitazone) & Takeda Pharmaceuticals ${ }^{d}$ & Activates peroxisome proliferator-activated receptor gamma & 1 \\
\hline & SRT2379 & GlaxoSmithKline & Sirtuin-1 activator & 1 \\
\hline
\end{tabular}

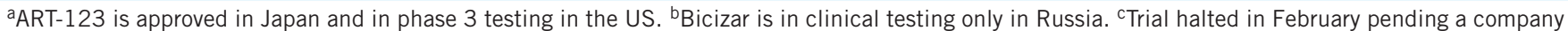
review of the data. ${ }^{d}$ Although the trial includes a Takeda drug, the company is not involved in the study. 